\title{
Regulation of Neuropeptide Stoichiometry in Neurosecretory Cells
}

\author{
Siegfried Hekimi, a Jacqueline Fischer-Lougheed, ${ }^{b}$ and Michael O'Shea \\ Interdisciplinary Research Centre for Neuroscience, School of Biological Sciences, University of Sussex, Falmer, Brighton, \\ Sussex BN1 9QG, England
}

\begin{abstract}
Peptidergic neurons and neurosecretory cells often contain multiple peptides, where they may be present in characteristic ratios. In this article, we describe how a set of five colocalized and coreleased peptides, two adipokinetic hormones (AKH I and AKH II), and three dimeric peptides (APRP 1,2 , and 3 ) are synthesized by the neurosecretory cells of the corpora cardiaca of the locust Schistocerca gregaria. We show that the five peptides are produced from two prohormones called pro-AKH I, or A-chain, and pro-AKH II, or $B$-chain. The amino acid sequences as determined by direct protein sequencing are given for both. Prior to processing, the two prohormones form the three possible dimers by the oxidation of the single cysteine residues found in each. The dimers, not the prohormones, are the direct precursors of the peptides. The dimeric precursors are called P1 (A-A), P2 (A-B), and P3 (B-B). Processing results in the generation of the two AKH peptides and the three dimers called adipokinetic hormone precursor-related peptides, or APRPs. Throughout postembryonic development, we show that the ratios of the AKHs and APRPs change dramatically and systematically. We show that these changes can be explained by the differential regulation of the synthesis of the two prohormones and their random association into dimers that are then completely processed. Regulation of peptide stoichiometry may expand the potential information content of the signals generated by multipeptide-producing neurons.
\end{abstract}

Many peptide-producing neurons and ncurosccretory cells generate multiple chemical signals by coproducing more than one peptide. The possibility arises, therefore, that an element of the chemical signal from such cells may be embodied in the ratios of the peptides produced. If peptide ratios represent signaling information, we may expect a strict regulation of peptide stoichiometry in multiple peptide-producing cells. An example of

\footnotetext{
Received Apr. 10, 1991; revised May 20, 1991; accepted May 22, 1991.

We are indebted to Elizabeth Fowler, William Burkhart, and Mary Moyer and to the Ciba Geigy Agriculture Biotechnology Research Unit for performing the amino acid sequence determination of the structures identified here. Dr. M. F. Schulz-Aellen provided critical input to the ideas expressed in this article. We thank Marianne Friedli for her technical assistance and Pat Hill, who prepared the manuscript. This work was supported by Ciba Geigy Ltd., the Swiss National Fund (Grant 3.181.0.85), the University of Geneva, and the Science and Engineering Research Council of the United Kingdom (Grant GR/E 86659).

Correspondence should be addressed to Michael O'Shea, Cell Biology Laboratory, University of London, New College (RHBNC), Egham, Surrey TW20 0EX, England.

- Present address: MRC Molecular Biology Laboratory, Hills Road, Cambridge, England $\mathrm{CB} 22 \mathrm{QH}$

'Present address: Laboratory of Neurobiology, University of Geneva, 1224 Chene Bougeries, Geneva, Switzerland.

Copyright (C) 1991 Society for Neuroscience $0270-6474 / 91 / 113246-11 \$ 05.00 / 0$
}

stoichiometry regulation has been found in the egg-laying hormone (ELH)-producing bag cells of Aplysia (Fisher et al., 1988). In these cells, ELH is represented once in a prohormone that also contains several other potential peptide signals. The stoichiometry of the peptides in the bag cells is, however, not the same as the stoichiometry of those peptides within the ELH precursor protein. The stoichiometry of the bag cell peptides appears to be regulated by a process requiring the differential degradation of a set of vesicles containing the non-ELH peptides. In this way, the ratios of released peptides are not predicted by the fixed stoichiometry of the peptides in the ELH precursor.

Here we describe a system of neurosecretory cells in which peptide stoichiometry changes systematically throughout postembryonic development. In spite of these changes, however, the peptide ratios are predicted by the fixed ratios of those peptides in their precursors. Differential regulation of peptide amounts is possible in this system because the peptides are contained in the structures of two colocalized prohormones whose synthesis is independently regulated. Additional flexibility of peptide stoichiometry is provided by the fact that the prohormones surprisingly are not the direct precursors of the peptides. Prior to processing, the prohormones are dimerized into two homo- and one heterodimer, and these three dimeric structures are the direct precursors. This allows the ratios of the monomer peptides (adipokinetic hormones, AKHs) to be altered at a different rate than the ratios of the dimer peptides (AKH precursorrelated peptides, APRPs) derived from the precursors. Here we provide, for the first time, the complete structures of the three dimeric precursors and the structures of all of their products of processing. These include the three dimer peptides called the APRP 1, -2, and -3. Our developmental studies show how the relative amounts of these peptides change throughout postembryonic development and how these changes can be explained by differential regulation of prohormone biosynthesis.

The neurosecretory cells of the corpora cardiaca (CC), major neuroendocrine organs of the desert locust Schistocerca gregar$i a$, produce, store, and release two neuropeptides with extensive sequence homology - the adipokinetic hormones (AKH I and AKH II) (Goldsworthy et al., 1972a,b; see Orchard, 1987, for review). Both are released into the circulation upon the initiation of flight behavior (Mayer and Candy, 1969), and they function to mobilize diacylglycerols from fat cells. In this way, they allow the animal to use lipid as an energy source during flight, thus permitting locusts to migrate great distances without feeding. AKH I is a 10-amino acid peptide, $p$ Glu-Leu-Asn-Phe-ThrPro-Asn-Trp-Gly-Thr- $\mathrm{NH}_{2}$ (Stone et al., 1976), and AKH II is 8 amino acids, $p$ Glu-Leu-Asn-Phe-Ser-Thr-Gly-Trp-NH (Sie- $_{2}$ gert et al., 1985). The CC consist of two distinct lobes, the storage and glandular lobes. The storage lobes are made primarily from the nerve endings of neurosecretory cells contained in the brain, 
to which they are connected by two paired nerves, and do not contain AKHs. By contrast, the glandular lobes contain an apparently uniform population of intrinsic neurosecretory cells (Rademakers and Beenakkers, 1977; Schooneveld et al., 1983; Hekimi and O'Shea, 1987) that produce both AKH I and AKH II in large amounts. Thus, the glandular lobes of the CC represent an $\mathrm{AKH}$ "peptide factory" much like the insulin-producing $\beta$-cells or the ELH-producing bag cells of Aplysia (see Sossin et al., 1989, for review). This feature of the glandular C. . coupled to the fact that they can be maintained easily in vitro, has made direct biochemical studies on the biosynthesis of the AKH neuropeptides and their precursors possible.

In a series of in vitro pulse-labeling experiments, two precursors of the AKHs called P1 and P2, both of about $8.4 \mathrm{kDa}$, were identified (Hekimi and O'Shea, 1987, 1989a). These experiments indicated that $\mathrm{Pl}$ is precursor to AKH I, the more abundant of the two hormones, and $\mathrm{P} 2$ is precursor to both $\mathrm{AKH} I$ and $\mathrm{AKH}$ II. When processed, P1 and P2 not only generate the AKHs but also two additional peptides that were named APRP 1 and APRP 2. The APRPs and the AKHs are released from the CC in a calcium-dependent manner when the neurosecretory cells are depolarized (Hekimi and O'Shea, 1989). The size of the APRPs (about $6.5 \mathrm{kDa}$ each) showed that they are major fragments of their precursors; indeed, it was clear from size considerations alone that $\mathrm{P} 1$ and $\mathrm{P} 2$ could accommodate only one copy of a $6.5 \mathrm{kDa}$ APRP and at most two AKH sequences each. Taken together, the evidence indicated that $P l$ consisted of two copies of AKH I (1 kDa each) and one copy of the $6.5 \mathrm{kDa}$ APRP 1, and P2 consisted of one AKH I, one AKH II $(0.8$ $\mathrm{kDa}$ ), and one copy of APRP 2. For P1, these structural predictions were subsequently confirmed by amino acid sequencing (Hekimi et al., 1989). The amino acid sequences and structures of P2, APRP 2, a third precursor (P3), and a third APRP have now been determined and are included in the Results.

The structure of Pl was determined following protein sequencing performed directly on the isolated and purified precursor (Hekimi et al., 1989). While the general structural features indicated from sizing experiments (two AKH I molecules and one APRP 1 per Pl) were confirmed by sequencing, one structural feature of the precursor Pl was entirely unexpected. The precursor $P 1$ is a dimer formed from two identical 41-residue subunits. Each subunit consists of one AKH I sequence (10 amino acids starting with pyroglutamic acid) followed by a canonical Gly-Lys-Arg processing site and then a 28 -residue carboxy-terminal peptide named the $\alpha$-chain containing a single cysteine. APRP 1 was shown to be a dimer of the 28-residue $\alpha$-chain. We call the subunit of the P1 precursor the A-chain. It is produced by a small mRNA (500 nucleotides) that encodes a very small 63-amino acid protein (prepro-AKH I) consisting of a 22-residue hydrophobic leader peptide followed by just one copy of the A-chain terminated by a stop codon (Schulz-Aellen et al., 1989). The A-chain is therefore by convention pro-AKH $I$, and we use the two terms interchangeably. This and additional evidence from in vitro translation experiments show that the homodimer Pl is made from independently translated A-chain monomers rather than from a larger prohormone containing more than one copy of the A-chain. Note that the AKH I prohormone (A-chain) is not the direct antecedent of AKH I because processing occurs in its dimeric form, P1. In this article, therefore, we draw a distinction between prohormone (the product of translation less the leader sequence) and precursor (the form of the prohormone that is processed).
This structural work on Pl provided the key to understanding the biosynthetic pathway for the complete complement of five peptides produced in the CC. In this article, as a prelude to developmental studies also reported here, we describe the formation, structure, and processing of the three dimeric precursors of the AKHs and APRPs. We show that like P1, P2 is a dimer consisting of one A-chain (pro-AKH I) and a different but homologous polypeptide we call the B-chain. The B-chain is proAKH II and consists of AKH II, a processing site followed by a 28 -residue carboxy-terminal sequence called the $\beta$-chain. This unusual molecular organization of dimeric precursors suggested the possible existence of an as yet unidentified third precursor (B-chain homodimer) and a third APRP ( $\beta$-chain homodimer). Here we demonstrate that the complete set of the three possible dimer precursors is synthesized in the CC. Using subunit-specific antisera, we also show that the A-chain and B-chain are found in the same cells and that all cells in the CC contain both. The two subunits are thus available to one another in all cells for dimer formation. Developmental studies show that systematic changes in the ratios of the AKH and APRP peptides occur throughcut postembryonic development. These changes can be predicted if we assume that independently translated $A$ - and B-chains (or pro-AKH I and pro-AKH II) associate randomly with one another producing ratios of dimeric precursors according to binomial distributions. Kinetic considerations localize the formation of dimer precursors to the endoplasmic reticulum. The random association model suggests that the changing peptide stoichiometries at different stages of development are produced by the differential regulation of the synthesis of the independently translated subunits alone.

\section{Materials and Methods}

Animals. Adult locusts (Schistocerca gregaria) used in this study were reared in our laboratory culture. CC dissected from more than 8000 individual locusts were used in this study. Female New Zealand White rabbits produced the antisera used for the immunocytochemical analysis.

Organ culture. Details of in vitro incubation methods were given in Hekimi and O'Shea (1987, 1989a) and Hekimi et al. (1989). Briefly, $\mathrm{CC}$ were removed from individual animals and incubated in the presence of radiolabeled $\left({ }^{3} \mathrm{H}\right.$ or $\left.{ }^{14} \mathrm{C}\right)$ amino acids in $100 \mu \mathrm{l}$ of physiological saline (see Results and figure captions for labeling protocols used in the various experiments). Up to $50 \mathrm{CC}$ can be incubated in this volume. Following varying durations of incubation with the labeled amino acid, the $\mathrm{CC}$ were either washed in saline and subjected to chromatography or transferred to a large volume of translation block medium in order to chase label from precursor to product. The translation block medium contained saline including $1 \mu \mathrm{g}$ per cycloheximide.

Extraction, identification, and purification procedures. Corpora cardiaca were homogenized in $100 \mu \mathrm{l}$ of $0.1 \%$ trifluoroacetic acid (TFA) at room temperature with an ultrasound cell disrupter. The TFA-soluble supernatant was removed and subjected to size-exclusion or reversephase chromatography. On-line detection was provided by a fixed wavelength absorbent detector set at $214 \mathrm{~nm}$. In some experiments, HPLC fractions were also subjected to scintillation counting. For size fractionation, a $60 \mathrm{~cm}$ TSK 2000 gel permeation column eluted at $0.5 \mathrm{ml} /$ min with $0.1 \%$ TFA containing $15 \%$ (vol/vol) acetonitrile was used. Complete details of procedures for calibrating the size-exclusion column have been provided previously (Hekimi and O'Shea, 1989a). Reversephase chromatography was achieved using a C8 wide-pore column ( 300 $\AA$ ) eluted at $1.5 \mathrm{ml} / \mathrm{min}$ with a linear gradient from $25 \%$ to $45 \%$ acetonitrile in $0.1 \%$ TFA at $1 \% / \mathrm{min}$. Criteria for the identification of peaks using synthetic peptides have been established previously (Hekimi and O'Shea, 1989).

Cleavage of the disulfide bonds between the subunits of the APRPs and Ps was obtained by reduction of peptides in $0.1 \mathrm{M}$ Tris- $\mathrm{HCl}, \mathrm{pH} 8$, with a 10 -fold excess of $\beta$-mercaptoethanol. Prior to sizing on the TSK 
Figure 1. Reverse-phase chromatography of the glandular lobes of the $\mathrm{CC}$ from adults $(A)$ and fourth larval stage $(B)$ locusts. The peptides were eluted using a linear gradient of acetonitrile in $0.1 \%$ TFA $(25-45 \%)$. Notice that, especially in the adult chromatogram, the pattern of OD peaks of this crude aqueous extract is extremely simple. The positions and optical densities of the peptides and precursors (except P3) referred to in this article are indicated. The more complex pattern of additional peaks found in $B$ is largely due to contamination from non-glandular lobe tissue that inevitably enters the extract because of the increased difficulty of dissecting pure glandular lobes from the relatively smaller $\mathrm{CC}$ from the earlier developmental stages. The differences in the stoichiometries of the peptides are apparent in comparing $A$ and $B$, especially for the $A P R P 1$ and $A P R P 2$ peaks.

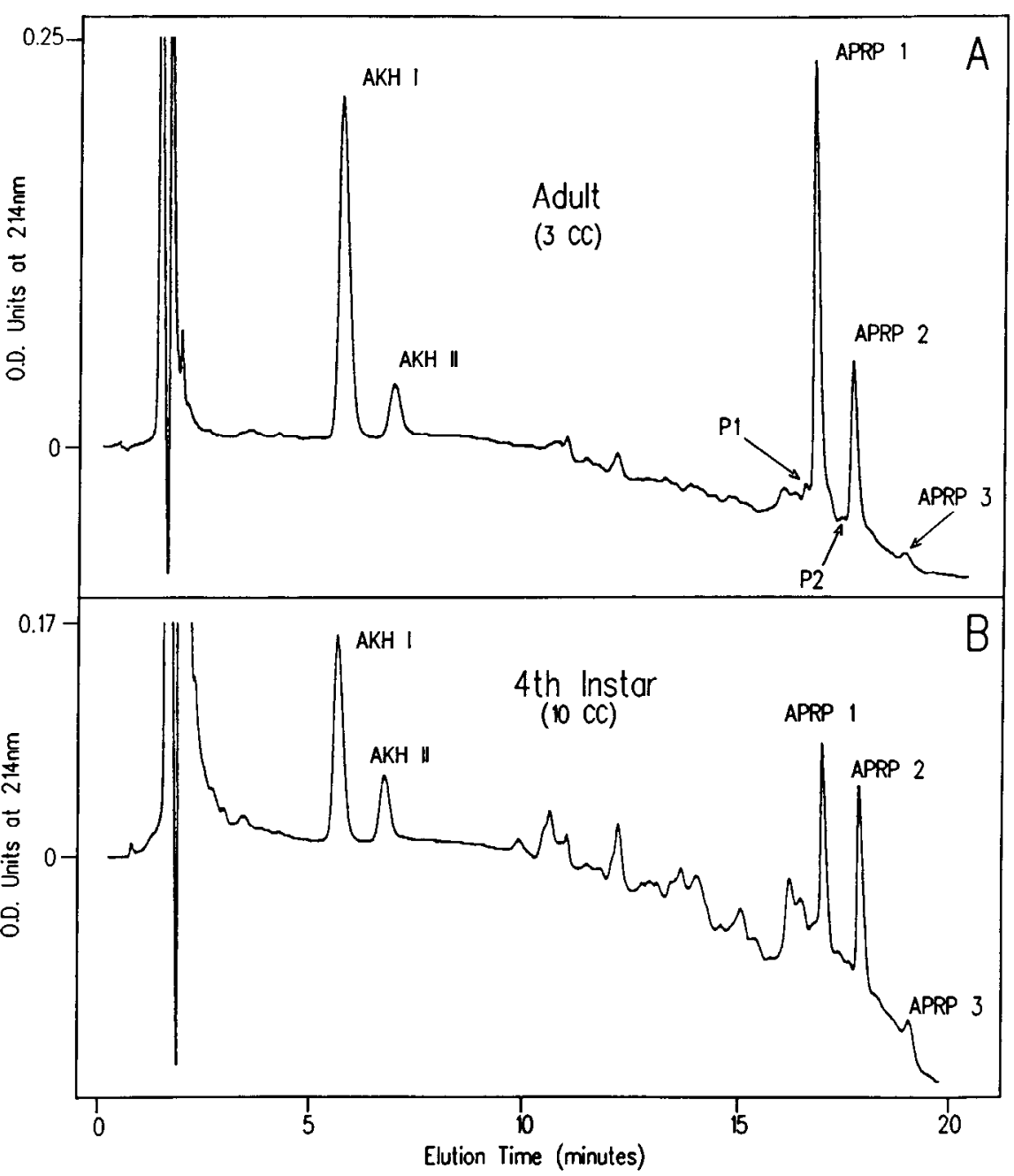

reactive material was revealed using 3,3'-diaminobenzidine (DAB; Fluka; $25 \mathrm{mg} \mathrm{DAB} / 100 \mathrm{ml} \mathrm{PB}+0.01 \% \mathrm{H}_{2} \mathrm{O}_{2}$ ). To demonstrate staining specificities, blocking experiments were performed by preincubating the antisera $1 \mathrm{hr}$ at room temperature in the dark with 5-10 $\mu \mathrm{M} \mathrm{AKH} \mathrm{I-}$ and $\mathrm{AKH}$ II-related antigens.

\section{Results}

The peptide content of the glandular cells of the $C C$

A crude aqueous extract of the neurosecretory cells of the CC shows a very simple pattern of optical density peaks when subjected to reverse-phase chromatography. The simplicity of this pattern of peaks reflects the homogeneity of the tissue with respect to neuropeptide synthesis. Figure 1 shows this pattern in a typical profile in which the positions of peptides referred to in this article are indicated. Notice that there are only four prominent optical density peaks, AKH I and AKH II and APRP 1 and APRP 2. The much smaller peaks attributed to the P1 and $P 2$ precursors migrate just ahead of the major APRP peaks. That P1 and P2 are precursors to the AKHs and APRP 1 and APRP 2 has been established by performing pulse-chase experiments on glands maintained in short-term tissue culture and provided with radiolabeled amino acids (Hekimi and O'Shea, 1987, 1989a). Structural analysis by protcin scquencing of P1 has confirmed its identity as precursor to two AKH I molecules and one APRP 1 (Hekimi et al., 1989). Previously reported pulse-chase experiments indicated that $\mathrm{P} 2$ was precursor to $\mathrm{AKH}$ I and AKH II and the $6.5 \mathrm{kDa}$ APRP 2. The complete primary 
structures of P2 and APRP 2 are determined in the present study, as are the identities and structures of a third precursor (P3) and a product (APRP 3).

The second chromatographic profile shown in Figure $1 B$ is from the $\mathrm{CC}$ of a pre-adult larval stage (fourth instar). While the same peptides are identifiable in the larval $\mathrm{CC}$, they occur in quite different relative amounts than in adults. For example, in the adult there is approximately a 2.5:1 ratio of APRP 1 to APRP 2, whereas in the fourth instar this ratio approaches $1: 1$. Similarly, the AKH I to AKH II ratios are also different in the adult $(\sim 5: 1)$ and the fourth instar $(\sim 3: 1)$. An explanation for these changing product stoichiometries is given below.

\section{Structure of APRP 2}

Our previous studies on APRP 1 suggested that APRP 2 may also be a dimer, so it was analyzed in both its reduced and native forms. Using size-exclusion HPLC, native APRP 2 is about 6.4 $\mathrm{kDa}$ but when reduced produces a single peak of $3.2 \mathrm{kDa}$. Both values are very similar to the size estimates achieved previously for native and reduced APRP 1 . When subjected to reversephase chromatography, reduced APRP 2 produces two optical density peaks of similar amplitude, whereas APRP 1, because it is a homodimer, produces a single peak. One of the subunits from reduced APRP 2 coelutes with the $\alpha$-chain subunit of APRP 1, the other having a longer retention time (Fig. 2). We call the second subunit of APRP 2 the $\beta$-chain. Size-exclusion chromatography does not allow us to distinguish between the $\alpha$ - and $\beta$-chains, indicating that they are of similar size $(\sim 3.2$ $\mathrm{kDa})$. That they can be separated by reverse-phase chromatography shows that the $\alpha$ - and $\beta$-chain subunits of APRP 2 are different peptides.

For sequence analysis, APRP 2 was reduced and alkylated, and the two subunits were subjected to amino acid sequencing following HPLC purification. The earlier-eluting peak gave, as expected, the complete sequence of the $\alpha$-chain only. The $\beta$-chain subunit produced a different, but related, 28 -residue sequence. Both sequences are given in Figure 3, in which the structure of APRP 2 as a disulfide-bridged heterodimer is given. In order to ensure that Edman sequencing was complete, both peptides present in reduced APRP 2 were subjected to hydrolysis followed by amino acid analysis. The amino acid compositions of the two peaks in APRP 2 (Table 1) indicated that the sequence analysis accounted for all the residues.

\section{Structure of P2}

Structural analysis of $\mathrm{P} 2$, presumptive precursor of AKH I, AKH II, and APRP 2, was more problematic primarily because it is present in very small amounts, less than half the level of $\mathrm{P} 1$. The low abundance of P2 meant that we could not easily monitor its optical density in the various experiments used to purify it and determine its size and structure. For monitoring, therefore, we used biosynthetically radiolabeled $\mathrm{P} 2$ produced by providing $\mathrm{CC}$ with ${ }^{3} \mathrm{H}$-Trp or ${ }^{14} \mathrm{C}$-Trp during in vitro biosynthesis.

Native P2 is about $8.4 \mathrm{kDa}$ (Hekimi and O'Shea, 1989a). When reduced and subjected to size-exclusion chromatography, biosynthetically labeled $\mathrm{P} 2$ shows a reduction in size to about half the native value, producing a single peak of label in gel filtration chromatography (data not shown). Using reverse-phase chromatography, however, reduced $\left[{ }^{14} \mathrm{C}-\mathrm{Trp}\right]-\mathrm{P} 2$ is resolved into two labeled peaks, one of which comigrates with the labeled A-chain derived from reduced $\left[{ }^{3} \mathrm{H}\right.$-Trp]-P1 (Fig. 4). These results are

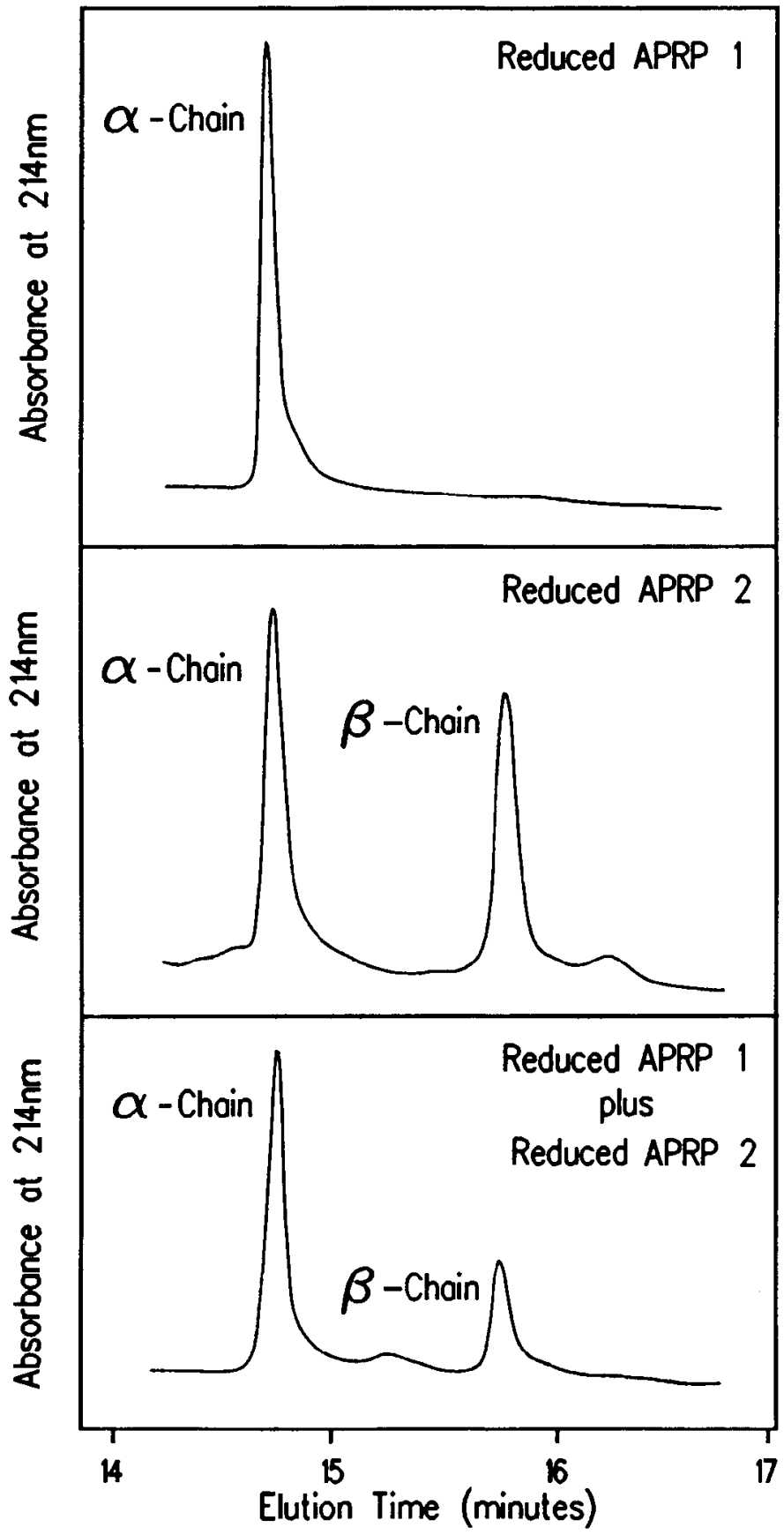

Figure 2. Reverse-phase chromatography of purified and reduced APRP 1 and APRP 2. The upper panel shows the single OD peak obtained from reduced APRP 1 . This peak has been shown previously by sequencing to be the 28-residue $\alpha$-chain. In the middle panel, reduced APRP 2 produces two peaks, one comigrating with the $\alpha$-chain of APRP 1. The two peaks generated from reduced APRP 2 have approximately equal areas, indicating subunits present in equimolar amounts. The later-eluting peak is shown in this article to be the 28-residue peptide called the $\beta$-chain. In the lower panel, reduced APRP 1 and reduced APRP 2 are mixed in equal amounts prior to reverse-phase separation. A peak comigrating with the $\alpha$-chain, which is approximately three times the integrated area of the peak comigrating with the $\beta$-chain, is produced. This would be expected from the proposed homodimeric structure of APRP 1 and heterodimeric structure of APRP 2. 
$\underline{\text { P1 }}$

A-chain (pro-AKH I)

pGlu-Leu-Asn-Phe-Thr-Pro-Asn-Trp-Gly-Thr Gly-Lys-Arg-Asp-Ala-Ala-Asp-Phe-Gly-Asp-Pro-Tyr-Ser-Phe-Leu-Tyr-Arg-Leu-Ile-Gin-Ala-Glu-Ala-Arg-Lys-Met-Ser-Gly-Cys-Ser-Asn-OH

APRP 1

pGlu-Leu-Asn-Phe-Thr-Pro-Asn-Trp-Gly-Thr -Gly-Lys-Arg-Asp-Ala-Ala-Asp-Phe-Gly-Asp-Pro-Tyr-Ser-Phe-Leu-Tyr-Arg-Leu-\|le-Gin-Ala-Glu-Ala-Arg-Lys-Met-Ser-Gly-Cys-Ser-Asn-OH

P2

$\alpha$-chain

PGlu-Leu-Asn-Phe-Thr-Pro-Asn-Trp-Gly-Thr -Gly-Lys-Arg Asp-Ala-Ala-Asp-Phe-Gly-Asp-Pro-Tyr-Ser-Phe-Leu-Tyr-Arg-Leu-lle-Gln-Ala-Glu-Ala-Arg-Lys-Met-Ser-Gly-Cys-Ser-Asn -OH

APRP 2

pGlu-Leu-Asn-Phe-Ser-Thr-Gly-Trp Gly-Arg-Arg Tyr-Ala-Asp-Pro-Asn-Ala-Asp-Pro-Met-Ala-Phe-Leu-Thr-Lys-Leu-lle-Gln-lle-Glu-Ala-Arg-Lys-Leu-Ser-Gly-Cys-Ser-Asn -OH

B-chain

P3

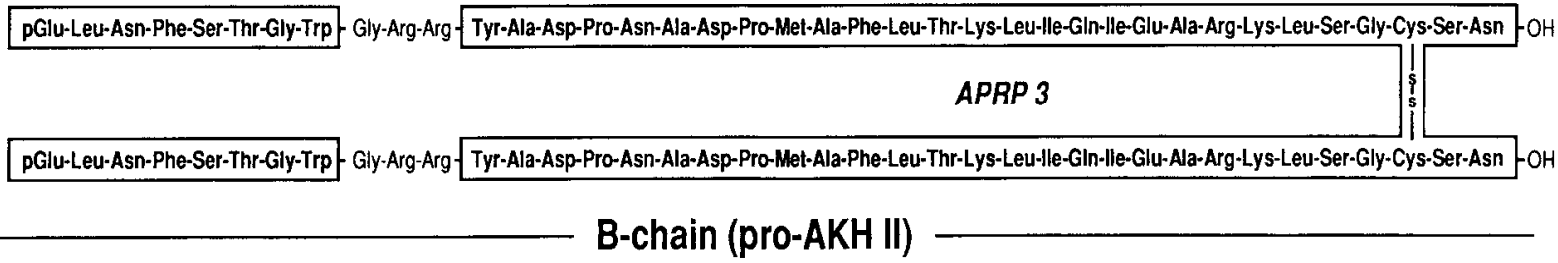

\section{AKHI}

pGlu-Leu-Asn-Phe-Thr-Pro-Asn-Trp-Gly-Thr -NH2
AKHII

pGlu-Leu-Asn-Phe-Ser-Thr-Gly-Trp-NH2

Figure 3. Structures of AKH precursors and products. Amino acid sequences and proposcd dimcric structures of the precursors and products (APRPs and AKHs) as described in this article are given. The presence of a single cysteine in the subunits and the behavior of the peptides when reduced indicate that a single disulfide bridge is formed in the synthesis of each precursor dimer. Notice that the A-and B-chains contain a potential dibasic (Arg-Lys) processing site in their respective $\alpha$ - and $\beta$-chain C-terminal peptides. These are not used in processing of the dimeric precursors in the CC.

consistent with $\mathrm{P} 2$ being a heterodimer formed by an A-chain and another subunit of similar size, presumably containing $\mathrm{AKH}$ II and the $\beta$-chain of APRP 2. To provide direct evidence for this, we undertook to sequence the second subunit of P2 by automated Edman degradation.

Attempts to purify P2 in sufficient quantity for sequencing proved impractical because separating it from APRP 2, with which it virtually comigrates, involved several steps of reversephase HPLC. Our efforts in this direction consumed approximately 5000 individually dissected $\mathrm{CC}$ and finally did yield a few picomoles of pure protein. Unfortunately, this amount, following further losses incurred during digestion with pyroglutamate aminopeptidase, was insufficient for sequencing. We therefore decided to sequence P2 (extracted from $3000 \mathrm{CC}$ ) following only one step of reverse-phase HPLC purification. Such a sample of $\mathrm{P} 2$ would generate a mixture of sequences because not only would it include $\Lambda$ PRP 2 , but P2 itself consists of two subunits. Following digestion with pyroglutamate aminopeptidase to remove amino-terminal pyroglutamate residues, the $\mathrm{P} 2$ preparation did reveal a mixture of sequences. The sequence for the A-chain subunit of P1 and the two subunits of APRP 2 were obtained as expected, as well as the as yet undetermined sequence of the second subunit contained in $\mathrm{P} 2$.
This sequence begins with the second residue of AKH II and continues through AKH II and a processing site into the sequence for the $\beta$-chain. From the inability to obtain sequence before digestion with pyroglutamate aminopeptidase and from the known sequence of AKH II that starts with pyroglutamic acid, we deduce that pyroglutamaic acid is at the amino terminus of the sequence. Sequencing of the second subunit of P2 continued through the first 13 residues of the $\beta$-chain before the signal became impossible to read. A protein sequencing ambiguity at the processing site was resolved by cDNA cloning (M.F. Schulz-Aellen, personal communication) and Arg-Arg is assigned for the B-chain, whereas for the A-chain the basic residues at this position are Lys-Arg. This sequencing data, together with the sizing evidence cited above and the known heterodimeric structure of APRP 2, indicated that P2 is a disulfide-bridged heterodimer formed from two prohormones, pro-AKH I or A-chain and pro-AKH II or B-chain (Fig. 3). Processing of the P2 heterodimer therefore yields AKH I and AKH II in equimolar amounts and one copy of APRP 2. Note that both the $A-$ and $B$-chains have a single dibasic processing site preceded by glycine. Glycine, as in other examples, provides the amidated C-terminal residue for AKH I and AKH II (Eipper et al., 1983, 1987). Interestingly, the dibasic site following glycine is different 
Table 1. Amino acid compositions of the $\alpha$ - and $\beta$-chains of the APRPs

\begin{tabular}{|c|c|c|c|c|}
\hline \multirow[b]{2}{*}{ Amino acid } & \multicolumn{2}{|l|}{$\alpha$-Chain } & \multicolumn{2}{|l|}{$\beta$-Chain } \\
\hline & $\begin{array}{l}\text { Moles aa/ } \\
\text { mole protein }\end{array}$ & $\begin{array}{l}\text { Residues/ } \\
\text { protein }\end{array}$ & $\begin{array}{l}\text { Moles aa/ } \\
\text { mole protein }\end{array}$ & $\begin{array}{l}\text { Residues/ } \\
\text { protein }\end{array}$ \\
\hline Aspartic acid & 2.85 & 4 & 2.04 & 2 \\
\hline Glutamic acid & 1.85 & 2 & 0.95 & 1 \\
\hline Serine & 2.80 & 3 & 2.94 & 3 \\
\hline Glycine & 2.05 & 2 & 1.32 & 1 \\
\hline Histidine & 0 & 0 & 0 & 0 \\
\hline Arginine & 2.20 & 2 & 1.23 & 1 \\
\hline Threonine & 0 & 0 & 0 & 0 \\
\hline Alanine & 4.18 & 4 & 4.22 & 4 \\
\hline Proline & 1.04 & 1 & 2.37 & 2 \\
\hline Tyrosine & 1.68 & 2 & 2.26 & 2 \\
\hline Valine & 0 & 0 & 0 & 0 \\
\hline Methionine & 0.74 & 1 & 0.79 & 1 \\
\hline Isoleucine & 1.52 & 1 & 1.59 & 2 \\
\hline Leucine & 2.07 & 2 & 2.75 & 3 \\
\hline Phenylaline & 1.91 & 2 & 0.88 & 1 \\
\hline Tryptophan & 0 & 0 & 0 & 0 \\
\hline Lysine & 0.95 & 1 & 1.57 & 2 \\
\hline Cysteine & Not determined & Not determined & & \\
\hline
\end{tabular}

aa, amino acid.

in two subunits, Lys-Arg for the A-chain and Arg-Arg for the B-chain. Also, a second but unused potential dibasic processing site exists (Arg-Lys) in both the 28-residue C-terminal $\alpha$ - and $\beta$-chains. The sequence for pro-AKH II or the B-chain of P2 as determined by protein sequencing has been fully confirmed by two independent cDNA cloning experiments in which the amino acid sequence was assigned by inference from a cloned cDNA (Noyes and Schaffer, 1990; Schulz-Aellen, personal communication). Although in many other systems cDNA cloning has provided a way to deduce prohormone structures, in this particular system it could not have predicted the actual dimeric nature of the direct precursors of the AKHs. The unusual properties of the $\mathrm{CC}$ have allowed us to perform direct structured experiments on the AKH precursors, and only in this way were their true dimeric structures assigned.

\section{Identification of a third dimeric AKH precursor (P3) and its cleavage product APRP 3}

The existence of a heterodimeric structure of $\mathrm{P} 2$ shows that proAKH I and pro-AKH II must, at least in some CC neurosecretory cells, exist in the same subcellular compartment. There is therefore no a priori reason why pro-AKH II or B-chains should not associate with one another to form a third precursor-a homodimer of pro-AKH II, P3 - which would be processed into two copies of AKH II and a $\beta$-chain homodimer, APRP 3.

With this in mind, we searched for previously unidentified components of the AKH biosynthetic pathway, P3 and APRP 3. That P3 and APRP 3 were not detected in our earlier studies suggested that they are relatively minor components of the system. We can now confirm that they do exist and that in the adult $\mathrm{CC}$ they are indeed minor components. Confirmation of their albeit meager existence, however, completes the posttranslational molecular model for AKH I and AKH II biosynthesis and has allowed us to understand the changing peptide stoichiometries in the $\mathrm{CC}$.

To identify P3, we used the pulse-labeling paradigm described previously for the identification and characterization of $\mathrm{P} 1$ and $\mathrm{P} 2$. We incubated $\mathrm{CC}$ in the presence of ${ }^{3} \mathrm{H}-\mathrm{Trp}$ and searched for proteins from which label can be chased into the tryptophan- containing AKHs. The chromatogram revealed a third but minor labeled peak from which the ${ }^{3} \mathrm{H}$-Trp disappeared during a chase experiment. This peak was purified and identified as the predicted third dimeric precursor (P3) by the following observations. In complete agreement with the expected behavior of the pro-AKH II homodimer, when reduced it produced a single labeled peak exactly comigrating with the B-chain derived from the reduction of $\mathrm{P} 2$. The existence of $\mathrm{P} 3$ is further supported by direct evidence for the production of a $\beta$-chain homodimer (APRP 3) in the CC. An optical density peak that is retained on the reverse-phase column even longer than APRP 2 (see Fig. 1) has been positively identified by protein sequencing as the third APRP with the expected $\beta$-chain homodimer structure. When reduced, APRP 3 produced a single peak of optical density comigrating in reverse-phase HPLC with the $\beta$-chain derived from APRP 2. Sufficient quantity of this peak was purified for protein sequencing, and this revealed only the complete primary structure of the $\beta$-chain. We propose that it is derived from P3, which when processed yields two AKH II molecules and one APRP 3.

Together, our experiments suggest a model for AKH biosynthesis in the CC according to which AKH I, AKH II, and three APRPs are generated by processing a complete set of three dimeric precursors produced from two prohormone subunits (Fig. 5). This biosynthetic scheme can provide an explanation for the relative abundance of each of the component proteins and peptides involved in neuropeptide biosynthesis in the $\mathrm{CC}$ (see below).

\section{Cellular colocalization is the rule}

The existence in the $\mathrm{CC}$ of the heterodimeric precursor $\mathrm{P} 2$ shows, as mentioned above, that at least some of the neurosecretory cells of the CC must synthesize both subunits. Such cells therefore may each contain all three dimeric precursors (P1, P2, and $\mathrm{P} 3$ ) in addition to all possible products (AKH I, AKH II, and the three APRPs). In order to link our molecular biological studies of AKH biosynthesis to the cell biology of the $\mathrm{CC}$, we needed to know if all AKH-expressing cells in the $\mathrm{CC}$ contain both subunits of the dimeric precursors. Antibodies with spec- 


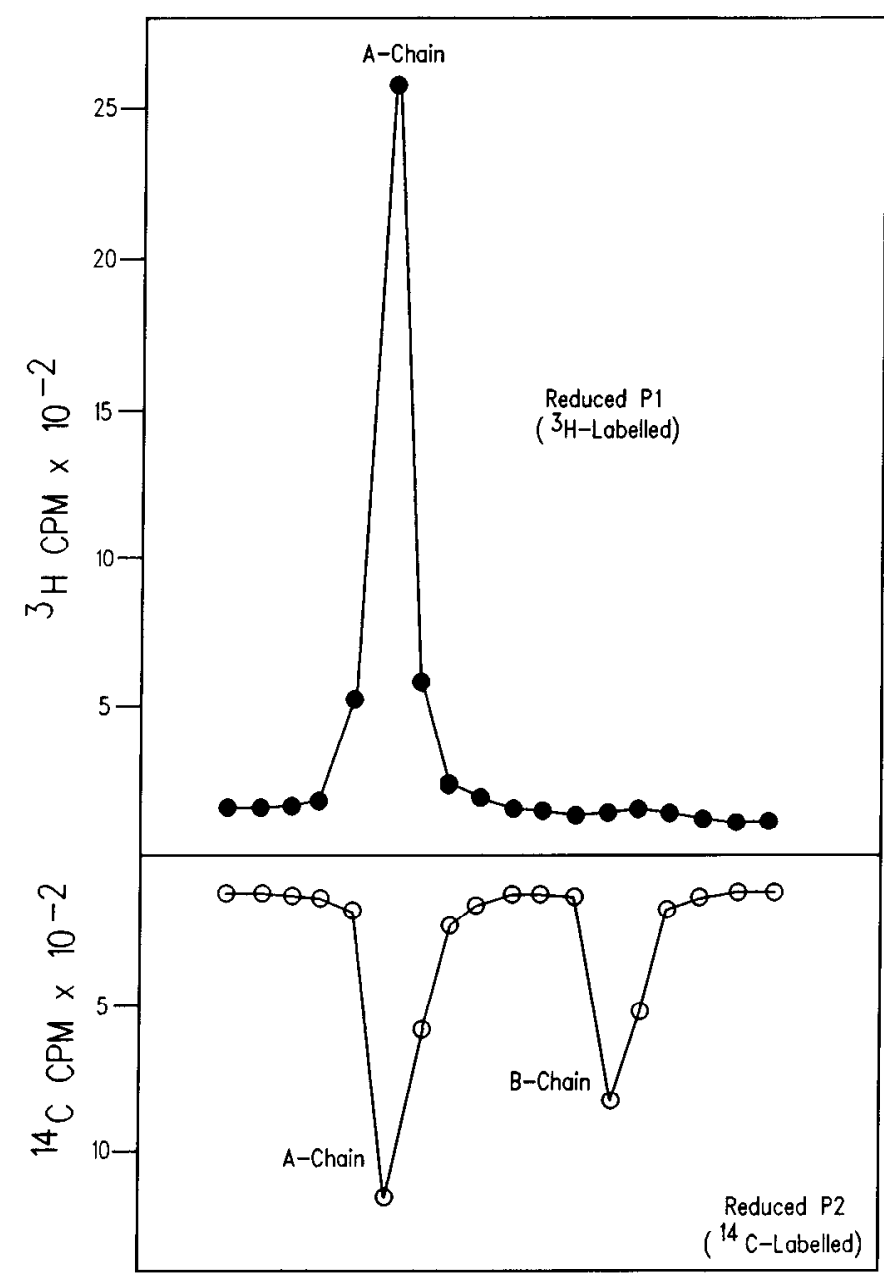

Fractions

Figure 4. Reversc-phase chromatography of biosynthetically labeled and reduced $\mathrm{P} 1$ and $\mathrm{P} 2$. The products of reduction were mixed prior to separation. The $\mathrm{P} 1$ was purified from $\mathrm{CC}$ incubated with ${ }^{3} \mathrm{H}-\mathrm{Trp}$ and biosynthetically labeled $\mathrm{P} 2$ was purified from glands incubated with ${ }^{14} \mathrm{C}$ Trp. Reduced P2 (lower panel) produces two peaks of label, one of which comigrates with the A-chain derived from reduced P1. This suggests that $\mathrm{P} 2$ is a heterodimeric construct. For further evidence and for the sequence of the B-chain, see Results and Fig. 3.

ificities designed to distinguish between pro-AKH I and proAKH II were therefore produced. These antibodies were raised against two synthetic oligopeptides that were designed to exploit the difference between AKH I and AKH II. The AKH I-specific antiserum was raised against Lys-Tyr[AKH I, 5-10], and the AKH II-specific serum was raised against Lys-Tyr[AKH II, 28]. The production and characterization of these antibodies that discriminate between AKH I and AKH II in a radioimmunoassay has been described previously (Hekimi and O'Shea, 1989b). For application in this study, we needed to demonstrate specificity of the immunoreactivity in immunocytochemistry by demonstrating differential blockade of staining following preabsorption with the oligopeptide antigens. Using these antisera in immunocytochemical experiments on the $\mathrm{CC}$, we have investigated whether all neurosecretory cells make both pro-AKH I and pro-AKH II or whether some cells exist that synthesize only one or the other.

Figure 6 shows that all immunoreactive cells in the $\mathrm{CC}$ are immunopositive for both AKH I and AKH II. It appears, there- fore, that all neurosecretory cells in the glandular lobes of the $\mathrm{CC}$ are able to synthesize both subunits of the three precursors. Since all cells appear to be equivalent, our molecular scheme derived from experiments performed on whole glands can represent events occurring in individual CC cells. Results of wholegland biochemical experiments might therefore legitimately be interpreted in terms of cellular and molecular events occurring in individual cells.

Random and rapid dimerization of prohormones followed by complete processing explains the characteristic stoichiometry of precursors and products

Pro-AKH I and pro-AKH II do not accumulate sufficiently to be reliably detected in our in vitro pulse-labeling experiments. When synthesis proceeds in vitro in the presence of ${ }^{3} \mathrm{H}-\mathrm{Trp}$, radiolabel can first be detected in dimerized precursors within a few minutes. It accumulates in the dimer precursors for 5-6 hr before reaching a steady-state level. The virtual absence of the monomer prohormones and the rapid appearance and accumulation of label in the dimer precursors indicate that dimerization occurs very close to the site of prohormone translocation into the endoplasmic reticulum. Indeed, dimerization may occur virtually cotranslocationally. In contrast to this very rapid posttranslational event, processing of the dimeric precursors to their products is delayed, as indicated by the delayed appearance of labeled AKH and by the accumulation of label in unprocessed precursors. During continuous incubation with ${ }^{3} \mathrm{H}-\mathrm{Trp}$, label in the AKHs begins to accumulate linearly when a steady-state level of label in the precursors is reached (Hekimi and O'Shea, 1987). These observations indicate that AKH biosynthesis involves the dimerization of prohormones in the endoplasmic reticulum followed by delayed transport and processing in another subcellular compartment (Fig. 5).

In the adult $\mathrm{CC}$, there is a striking and characteristic imbalance in the relative amounts and rates of synthesis of dimeric precursors and their monomeric and dimeric products. The largest imbalance is between the absolute level of precursors and products. A single $\mathrm{CC}$ from mature adult can contain as much as $1 \mathrm{nmol}$ of AKH I. The dimeric precursors, however, because they are metabolic intermediates, are present in far lower levels (picomolar and subpicomolar levels per CC) and are difficult or, in the case of P3, impossible to measure by their optical density. More interesting than the relatively low abundance of precursors compared to products are the changing relative levels of the various components of the biosynthetic pathway. For example, in the adult, the synthesis of $\mathrm{P} 1$ proceeds at about twice the rate of $\mathrm{P} 2$, and $\mathrm{P} 3$ synthesis is virtually nil by comparison. Related to these differences in precursor synthesis rates, the product ratios also show characteristic imbalances. The adult AKH I to AKH II ratio is almost 5:1, and the APRP 1 to APRP 2 ratio is more than 2:1, with relatively little APRP 3 . Some of these differences in product ratios are apparent in Figure 1.

These differing ratios can be explained by our molecular model (Fig. 5) if we make some simple assumptions. Consider, for example, a situation where the precursor subunits, pro-AKH I and pro-AKH II, are available for dimer formation in equal amounts. Assuming random dimerization with no preferential association, there would be a perfect binomial distribution (1: $2: 1$ ) of the dimeric precursors $\mathrm{P} 1: \mathrm{P} 2: \mathrm{P} 3$. If we also assume complete processing of precursors, this would lead to an AKH I to AKH II ratio of $1: 1$ and a binomial distribution of the APRPs of $1: 2: 1$. Any differences in the relative availability of the sub- 

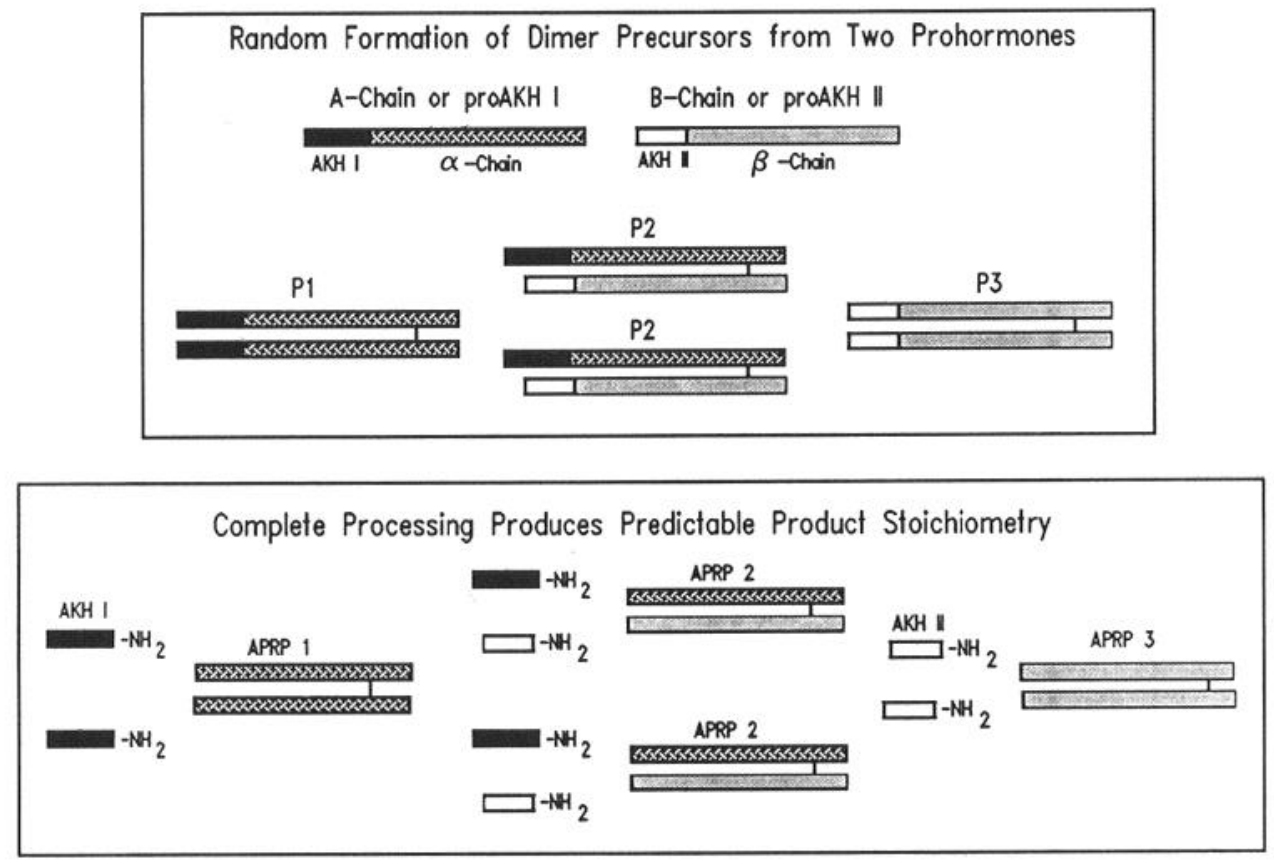

Figure 5. A scheme illustrating the posttranslational events from the dimerization of pro-AKH I and pro-AKH II into the three dimeric precursors and their subsequent processing to the three APRPs and the two AKHs. For further explanation and for an explanation of how this model can predict the changing product stoichiometries, see Discussion.

units would be reflected in skewed binomial distributions of precursors and products. These ideas could be tested experimentally if we were able to estimate the relative rates of synthesis of the two prohormones. We are unable, however, to measure directly the relative rates of synthesis of the A- and B-chains because, as indicated above, dimerization is very rapid and the first sign of AKH biosynthesis is label appearing in the accumulating dimeric precursors. Since, however, the A-chain contains one copy of AKH I and the B-chain contains one copy of $\mathrm{AKH}$ II, a reflection of their relative rates of synthesis can be obtained from measuring the relative amounts of AKH I and AKH II produced. In the adult $\mathrm{CC}$, the relative proportion of AKH I to AKH II approaches 5:1, suggesting that there may be almost five times as much A-chain as B-chain translated. Assuming a 5:1 ratio of $\mathrm{A}$-chain to $\mathrm{B}$-chain and assuming random association, there would be a skewed binomial distribution of $\mathrm{P} 1: \mathrm{P} 2: \mathrm{P} 3$ producing ratios of $25: 10: 1$. Furthermore, if the precursors in these ratios are all completely processed, a 5:1 ratio of $\mathrm{AKH}$ I to AKH II would be accompanied by a 2.5:1 ratio between APRP 1 and APRP 2 (with APRP 3 being $1 / 25$ th of APRP 1). Ratios very close to these relationships are found in the adult $\mathrm{CC}$, so our assumptions concerning the free random association of A- and B-chains and their complete processing may be valid. We suggest, therefore, that the ratios of the AKHs and APRPs are determined by the relative synthesis rates of the two prohormones alone. An opportunity to test this hypothesis is provided by studies on the $\mathrm{CC}$ at different stages of postembryonic development during which product stoichiometries change systematically.

\section{Developmental regulation of synthesis}

During postembryonic development, lasting about 5 weeks, the locust passes through five larval stages or instars before moulting to the winged adult. Throughout this period, the CC grow and absolute amounts of the AKHs and APRPs increase dramatically. For example, adult levels of AKH I are approximately 500 -fold greater than in the first larval stage or first instar. Increase of absolute amounts of peptide is relatively greater than the rate of growth of the organism as a whole, and this results in increased peptide levels when amounts are normalized to body weight. While levels of all CC peptides increase during development, they do not increase at equal rates. For example, the increase in AKH I during postembryonic development is greater than the increase in AKH II, and this produces systematic changes in the relative amounts or ratios of the peptides (Fig. 7). Notice that in the first larval stage the AKH I to AKH II ratio is close to $1: 1$, whereas in the adult the ratio approaches $5: 1$, and also that the normalized levels of both AKH I and AKH II increase abruptly in late postembryonic development and in the adult. This latter feature is interesting because the AKHs are involved in regulating lipid metabolism during flight and flight is an entirely adult behavior. During the last two postembryonic transitions (from fourth to fifth and from fifth to adult), there is a partial metamorphosis involving the rapid development of wings. Metamorphosis is known in insects to be associated with a fall in the titer of juvenile hormone, so this hormonal change may also trigger the upregulation of $\mathrm{AKH}$ biosynthesis.

At the same time that the AKH ratios change, the APRP ratios also change systematically. Indeed, the changes in the APRP ratios are even more pronounced. For example, in the first larval stage the APRP 1 to APRP 2 ratio is approximately $0.5: 1$, and in the adult, approximately $2: 1$. A comparison between the postembryonic developmental changes in the relative amounts of the AKHs and APRP 1 and APRP 2 is shown in Figure 8. Notice that the developmental change in the $\mathrm{AKH}$ ratio is less than the change in the APRP ratio by a factor of approximately 2 . Thus, for the whole period of postembryonic development, the AKH ratio changes by about 2.7-fold, whereas the APRP ratio changes by approximately 4.7 -fold.

How can these complex changes in peptide relative levels and ratios be simply explained? Our data suggest strongly that the explanation is to be found in the changing relative rates of synthesis of the two prohormones, the A- and B-chains. This being so, we ought to be able to show a predictable correlation between relative rates of precursor synthesis and the relative 


\section{Control}

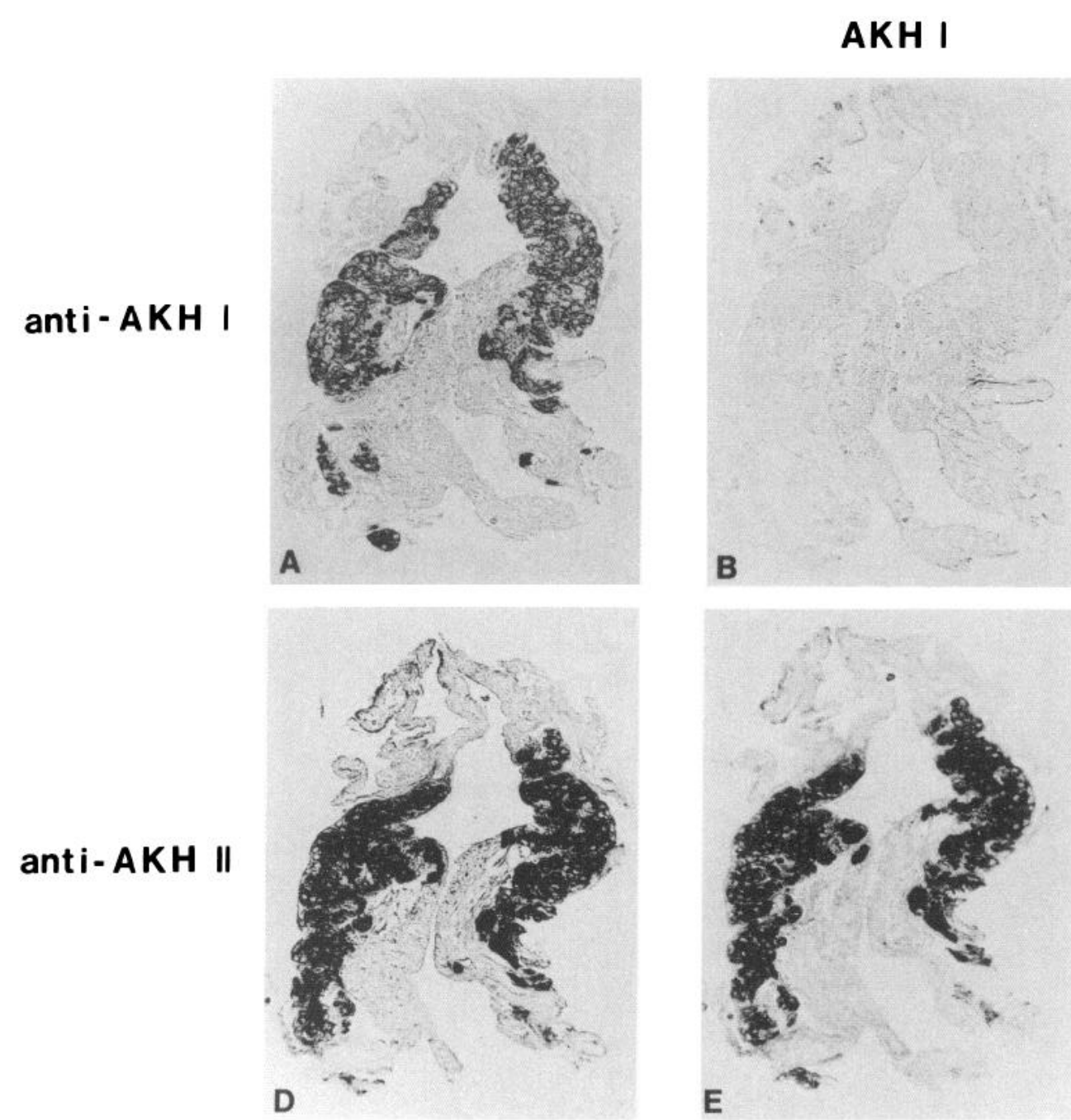

\section{Preabsorbed with}
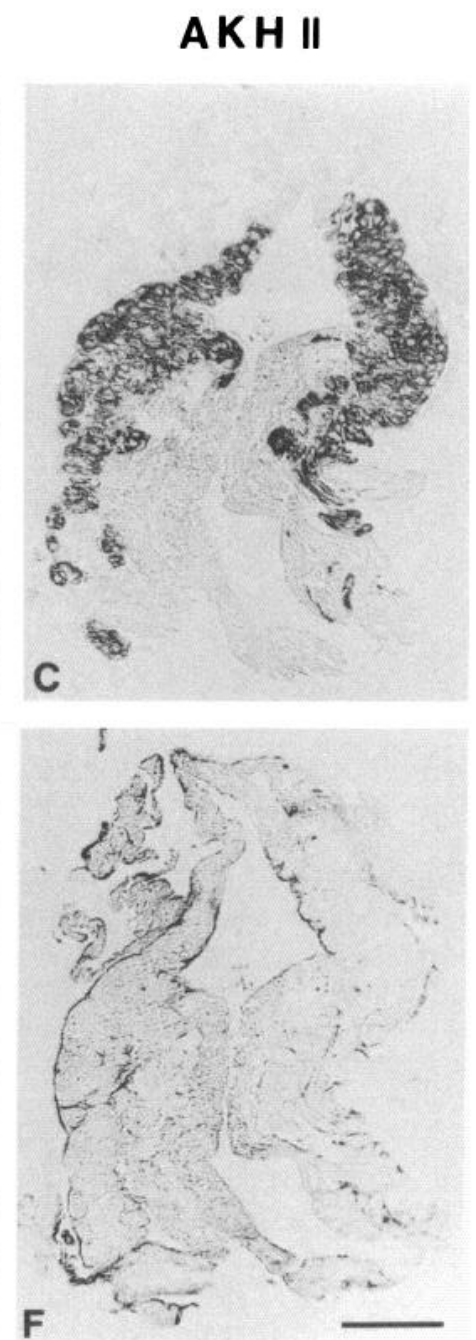

Figure 6. Immunocytochemical evidence for the colocalization of two prohormones (pro-AKH I, or the A-chain, and pro-AKH II, or the B-chain) in the glandular neurosecretory cells of the CC. In $A$, staining with an anti-AKH I-specific antibody reveals the neurosecretory cells of the CC. Specificity is indicated in $B$ and $C$ by the demonstration of a blockade of staining when the antibody is preincubated with AKH I, but not with AKH II. In the lower panels, a similar series of sections demonstrates the presence of AKH II in the neurosecretory cells of the CC. In these panels, staining blockade occurs when the antibody is preabsorbed with AKH II $(F)$, but not with AKH I $(E)$. Scale bar, $100 \mu \mathrm{m}$.

amounts of processed peptides at different stages of development. Precursor synthesis can be measured at different stages of postembryonic development by comparing the incorporation of the ${ }^{3} \mathrm{H}$-Trp into P1 and P2 (the two most easily monitored precursors) during precursor synthesis in vitro. As each prohormone contains the same number of tryptophan residues, the amount of tritium coeluting with $\mathrm{P} 1$ and $\mathrm{P} 2$ indicates the relative synthesis rates of the precursors. As expected from the measurements of AKH synthesis throughout development, there are changes in the relative rates of $\mathrm{P} 1$ and $\mathrm{P} 2$ synthesis as a function of stage of postembryonic development. For example, in the first instar CC the $\mathrm{P} 1$ to $\mathrm{P} 2$ ratio is approximately $0.5: 1$, whereas in the adult it is more than $2: 1$. These results suggest that the changes in the pattern of neuropeptide stoichiometry are being driven by developmentally regulated changes in the rates of precursor biosynthesis. Our molecular model for the biosynthesis of AKH I and AKH II (Fig. 5) can be used to make specific predictions about the expected relative amounts of $\mathrm{AKH} \mathrm{I}$ and
AKH II and the APRPs when the rates of synthesis of the precursors are altered. Assuming that the two subunits of the precursors associate randomly into the dimers that are then completely processed, we can calculate the expected AKH I to AKH II and APRP 1 to APRP 2 ratios from the P1:P2 synthesis ratio alone. For example, since $\mathrm{P} 1$ and $\mathrm{P} 2$ each contain one copy of an APRP, the slope of the relationship between the P1 to P2 ratio and the APRP 1 to APRP 2 ratio should be 1 . Since, however, there is twice as much $\mathrm{AKH} \mathrm{I}$ in P1 as in P2, the relationship between a changing precursor ratio (P1:P2) and the AKH I to AKH II ratio should have a slope of $1 / 2$. Notice that from this we would expect the APRP 1 to APRP 2 ratio to change at twice the rate of the AKH ratio during development. A twofold change in the AKH ratio would therefore be associated with a fourfold change in the APRP ratios. This expected association is close to the experimentally measured difference between changing AKH and APRP ratios, as illustrated in Figure 8 . 


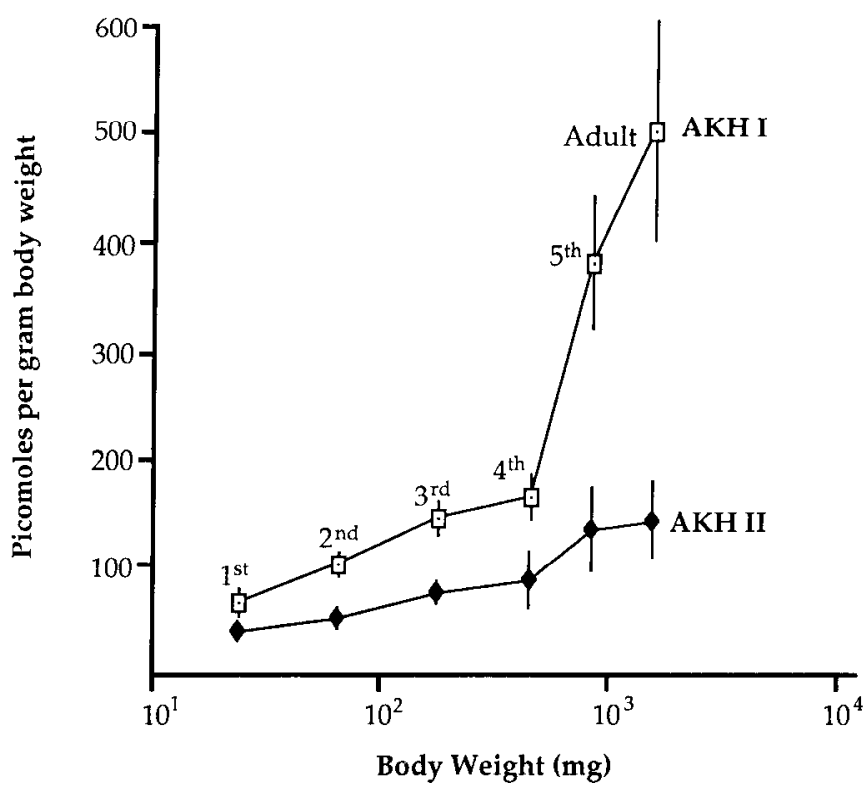

Figure 7. Postembryonic developmental changes in the amounts of AKH I and AKH II per CC normalized to body weight. Levels of both peptides increase throughout postembryonic development, but the increase in $\mathrm{AKH}$ II is greater than the increase in $\mathrm{AKH} \mathrm{I}$, producing progressive shifts in the ratios between the two peptides. The developmental changes in these ratios are plotted in Figure 8, in comparison to developmental changes in APRP ratios. The numbers beside the data points $\left(I^{s t}-5^{t h}\right)$ indicate the postembryonic larval stages and development. The error bars indicate $\pm 1 \mathrm{SD}$.

\section{Discussion}

\section{A test of the biosynthetic model}

As a test of our hypothesis and to determine whether changing peptide stoichiometries were driven by changing relative rates of precursor biosynthesis, we examined the relationship throughout postembryonic development between the experimentally determined rates of $\mathrm{P} 1$ and $\mathrm{P} 2$ synthesis and the experimentally determined amounts of the peptides present. Figure 9 shows that these experimentally derived data points fall close to the two slopes theoretically derived from binomial calculations based on the assumption of random association of two prohormones. The close correspondence between observed and predicted ratios suggests that our molecular model and the assumptions related to it are largely valid. In conclusion, the changes in neuropeptide ratios seen in postembryonic development can most simply be explained by the differential regulation of the synthesis of the two prohormones, the A- and B-chain subunits of the dimeric precursors. We do not know precisely how regulation of the subunit of biosynthesis is achieved, but in general terms this may be either largely transcriptional or translational. Whatever the mechanism, however, it is clear that the CC show strong differential control on neuropeptide precursor biosynthesis and this results in complex but systematic and expected changes in neuropeptide stoichiometry.

\section{Significance of dimeric precursors and the changing peptide ratios}

Perhaps there is signaling information contained in peptide ratios. It is appealing to think that information could be encoded in neuropeptide ratios because unlike the absolute levels of peptides, which might be more difficult to regulate, ratios can be regulated simply by changing relative synthesis rates of precur-

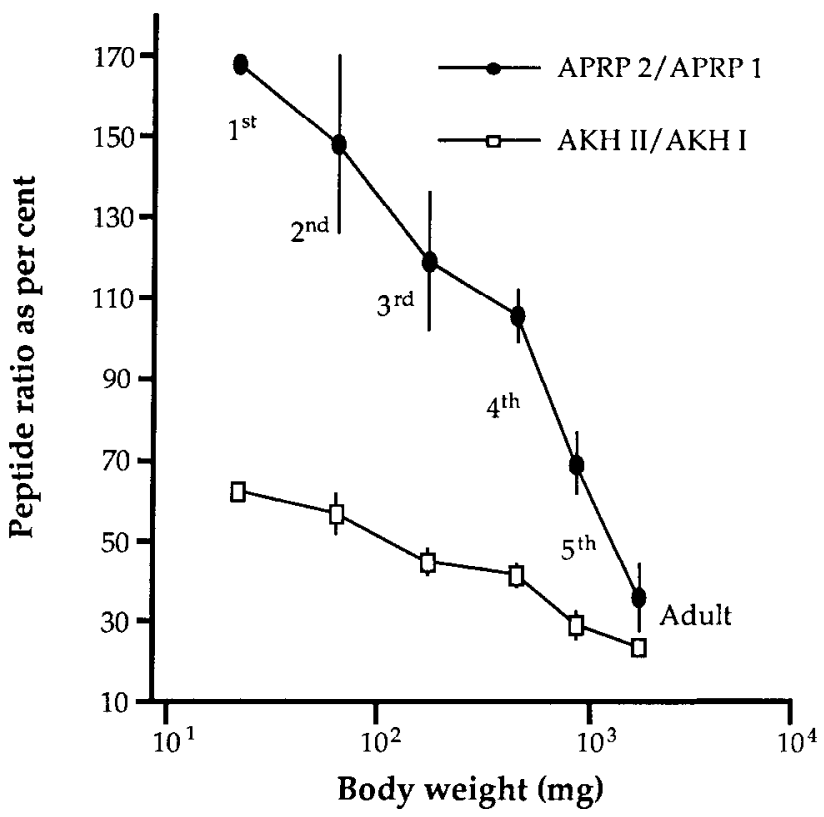

Figure 8. Changes in peptide ratios (AKH II to AKH I and APRP 2 to APRP 1) during postembryonic development. The numbers beside the data points $\left(l^{\mathrm{s}}-5^{\prime h}\right)$ indicate the postembryonic larval stages of development. The error bars indicate $\pm 1 \mathrm{SD}$.

sors. The ratios therefore could contain signaling information that would be unaffected by nonspecific factors such as nutrition and health, which might alter absolute peptide levels. Although we do not yet know the function of the APRPs, they are corelcascd with the AKHs (Hekimi and O'Shea, 1989a) and their amino acid sequences are highly conserved in distantly related locust species (S. Hekimi, I. Fischer-Lougheed, and M. O'Shea, unpublished observations). This suggests they also have signaling or hormonal roles. In spite of our incomplete understanding of the role of the APRPs, it seems unreasonable to assume that there is no biological significance to the changing ratios, especially since these appear to be so precisely, progressively, and systematically varied during development.

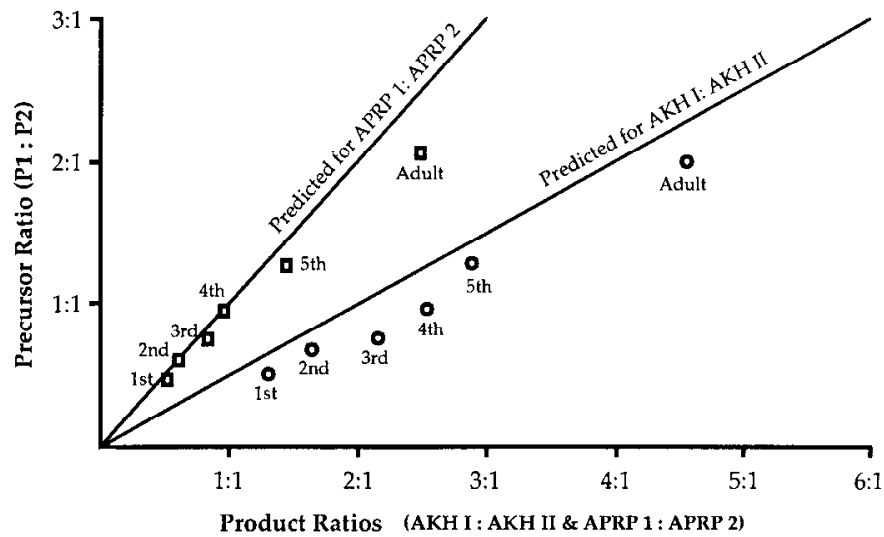

Figure 9. Relationship between relative rates of precursor synthesis (P1 to $\mathrm{P} 2$ ) and the relative amounts of products (AKH I to AKH II and APRP 1 to APRP 2) throughout postembryonic development. The slopes of these relationships, as predicted from our molecular model and our assumptions about it, are shown as solid lines. The experimentally derived data points are shown as squares (for the APRP ratios) and circles (for the AKH ratios). There is good agreement between the predicted and observed data, and an explanation for this is provided in the Discussion. 
The special feature of the biosynthetic system of the CC that allows for changes in peptide stoichiometry is the coexpression of two different prohormones. Cells producing multiple peptides in which only one prohormone is expressed can also vary the product ratios, but in such systems mechanisms involving segregation, sorting, and differential degradation are required (Fisher et al., 1988; Sossin et al., 1990a,b). By processing multiple colocalized peptides from more than one prohormone, the neurosecretory cells of the $\mathrm{CC}$ can simply regulate the relative levels of peptides by differential regulation of precursor synthesis. Moreover, by forming three dimer precursors from the two prohormones, the ratios of different types of peptides (monomers and dimers) change at different rates. All of this would seem to expand greatly the potential signaling capability of multiple peptide cells. If similar phenomena occur in multipeptide neurons of the CNS, the information content of a neuronal chemical signal may be significantly enhanced.

\section{References}

Bidlingmeyer BA, Cohen SA, Tarvin TL (1984) Rapid analysis of amino acids using pre-column derivatization. J Chrom Biomed Appl 336:93-104.

Eipper BA, Mains RE, Glembotski CC (1983) Identification in pituitary tissue of a peptide $\alpha$-amidation activity that acts on glycineextended peptide and requires molecular oxygen, copper, and ascorbic acid. Proc Natl Acad Sci USA 80:5144-5146.

Eipper BA, Park LP, Dickerson IM, Keutmann HT, Thiele EA, Rodriguez H, Schofield PR, Mains RE (1987) Structure of the precursor to an enzyme mediating $\mathrm{COOH}$ terminal amidation in peptide biosynthesis. Mol Endocrinol 1:777-790.

Fisher JM, Sossin W, Newcomb R, Scheller RH (1988) Multiple neuropeptides derived from a common precursor are differentially packaged and transported. Cell 54:813-822.

Goldsworthy GJ, Johnson RA, Mordue W (1972a) In vivo studies on the release of hormones from the corpora cardiaca of locusts. J Comp Physiol 79:85-96.

Goldsworthy GJ, Mordue W, Guthkelch J (1972b) Studies on insect adipokinetic hormones. Gen Comp Endocrinol 18:545-551.

Hekimi S, O'Shea M (1987) Identification and purification of two precursors of the insect neuropeptide adipokinetic hormone. J Neurosci 7:2773-2783
Hekimi S, O'Shea M (1989a) Biosynthesis of adipokinetic hormones (AKHs): further characterization of precursors and identification of novel products of processing. J Neurosci 9:996--1003.

Hekimi S, O'Shea M (1989b) Antisera against AKHs and AKH precursors for experimental studies of an insect neurosecretory system. Insect Biochem 19:79-83.

Ilekimi S, Burkhart W, Moyer M, Fowler E, O'Shea M (1989) Dimer structure of a neuropeptide precursor established: consequences for processing. Neuron 2:1369-1373.

Mayer RJ, Candy DJ (1969) Control of haemolymph lipid concentration during locust flight: an adipokinetic hormone from the corpora cardiaca. J Insect Physiol 15:611-620.

Noyes BE, Schaffer MH (1990) The structurally similar neuropeptides adipokinetic hormone I and II are derived from similar, very small mRNAs. J Biol Chem 265:483-489.

Orchard I (1987) Review: adipokinetic hormones: an update. J Insect Physiol 33:451-463.

Rademakers LHP, Beenakkers AMT (1977) Changes in the secretory activity of the glandular lobe of the corpus cardiacum of Locusta migratoria induced by flight. A quantitative electron microscope study. Cell Tissue Res 180:155-171.

Schooneveld H, Tesser GI, Veenstra J, Romberg-Privee HM (1983) Adipokinetic hormone and AKH-like peptide demonstrated in the corpora cardiaca and nervous system of Locusta migratoria by immunocytochemistry. Cell Tissue Res 230:67-76.

Schulz-Aellen M-F, Roulet E, Fischer-Lougheed J, O'Shea M (1989) Synthesis of a homodimer neurohormone precursor of locust adipokinetic hormone studied by in vitro translation and cDNA cloning. Neuron 2:1369-1373.

Siegert KJ, Morgan P, Mordue W (1985) Primary structures of locust adipokinetic hormones II. Hoppe Seylers Z Physiol Chem 366:723727.

Sossin WS, Fisher JM, Scheller RH (1989) Cellular and molecular biology of neuropeptide processing and packaging. Neuron 2:14071417.

Sossin WS, Fisher JM, Scheller RH (1990a) Sorting within the regulated secretory pathway occurs in the trans-Golgi network. J Cell Biol 110:1-12.

Sossin WS, Sweet-Cordero A, Scheller RH (1990b) Dale's hypothesis revisited: different neuropeptides derived from a common prohormone are targeted to different processes. Proc Natl Acad Sci USA 87: 4845-4848.

Stone JV, Mordue W, Batley KE, Morris HR (1976) Structure of locust adipokinetic hormone that regulates lipid utilisation during flight. Nature 263:207-211. 